\title{
EFFECT OF CROSS BREEDING BETWEEN PEKIN AND SUDANI (EGYPTIAN MUSCOVY) DUCK ON PHENOTYPIC PARAMETERS
}

\author{
A. Makram ${ }^{1}$, A. Galal ${ }^{2}$ and A.H. El-Attar ${ }^{2}$ \\ 1- Poultry Production Department, Faculty of Agriculture, Fayoum University, Egypt, 2-Poultry Production \\ Department, Faculty of Agriculture, Ain Shams University, Cairo, Egypt, Corresponding author: Amer Makram Ali *. \\ E-mail: Am150@Fayoum.edu.eg
}

Sudani duck is a native strain in Egypt. They have lower growth performance, although, Sudani ducks have higher carcass quality and immune response. On the other hand ، Pekin duck grow rapidly, reaching approximately $90 \%$ of their adult weight at 7 weeks of age. The objective of this study improving productive performance for Sudani duck by crossing its females Pekin males. At 32 wks of age, the Pekin (P) males allow to natural mating with Sudani (S) female. Total number duckling was 125, 150 and 74 for Pekin Sudani and the cross between Pekin with Sudani ducks (PS Cross) respectively. Body weight was recorded according to the marketing age ( 8,9 and 14 wks for the Pekin, PS cross, and Sudani ducks, respectively), body weight gain; growth rate and feed conversion ratio were calculated weekly. Body measurements were measured from the second week of the age. The crossbreed effect or heterosis (Hybrid vigor) was calculated. Results showed that the PS cross had significantly heaviest body weight compared to their parents at all ages. Concerning feed conversion ratio, Sudani and Pekin duck had better feed conversion ratio compared to their cross from 2-4, 6-8 and 2-8 weeks of age. The PS cross had significantly higher body measurements at 2, 4, 6 and 8 weeks of age compared to Sudani and Pekin one. The PS cross recorded negative heterosis at one day old of body weight, however, there was positive heterosis at all ages. With respect to growth rate, the heterosis was positive at all ages except from 2-3 and 7-8 wks of the age, however, the high positive value recorded from 5-6 wks of age was 91\%.Conclusion: In conclusion, the PS cross exhibited a higher figure in growth performance compared to Sudani duck. So it can be used as a commercial hybrid.

Keywords: Phenotypic parameters, Growth parameters, Body Measurements, Sudani, Pekin duck, Cross, Heterosis. 